\title{
Parkinson Kinetic Graph: Are Motor Fluctuations in Parkinson Disease Related with Disease Duration?
}

\section{Esther Berghuis ${ }^{1}$, Barbera Van Harten1, Mirjam Van Kesteren-Biegstraaten ${ }^{2}$, Wijnand Rutgers ${ }^{3}$, Nicolaas Verwey ${ }^{*}$}

\author{
${ }^{1}$ Department of Neurology, Medisch Centrum Leeuwarden, Leeuwarden, The Netherlands \\ ${ }^{2}$ Department of Neurology, Isala Zwolle, Zwolle, The Netherlands \\ ${ }^{3}$ Department of Neurology, Martini Ziekenhuis Groningen, Groningen, The Netherlands \\ Email: *niek.verwey@znb.nl
}

How to cite this paper: Berghuis, E., Van Harten, B., Van Kesteren-Biegstraaten, M., Rutgers, W. and Verwey, N. (2018) Parkinson Kinetic Graph: Are Motor Fluctuations in Parkinson Disease Related with Disease Duration? Advances in Parkinson's Disease, 7, 1-6.

https://doi.org/10.4236/apd.2018.71001

Received: November 22, 2017

Accepted: January 28, 2018

Published: January 31, 2018

Copyright $\odot 2018$ by authors and Scientific Research Publishing Inc. This work is licensed under the Creative Commons Attribution International License (CC BY 4.0).

http://creativecommons.org/licenses/by/4.0/

\begin{abstract}
Introduction: The Parkinson's KinetiGraph (PKG) is a digital measurement system for motor fluctuations (FS). The aim of this study is to investigate whether FS, measured by the PKG, are associated with disease duration of Parkinson's Disease (PD) patients. Material and Methods: 172 PD were included. PKG measurements, clinical data and disease duration were collected. Patients were categorized in four disease duration categories ( 0 - 5 years, 6 - 7 years, 8 - 11 years and $\geq 12$ years). Kruskal-Wallis and Mann-Whitney $U$ tests were used for statistical analysis. Results: The mean age of the patients is 69.2 years ( $S D \pm 8.13)$. Disease duration varies between 1 and 28 years. Significant difference was found between the four disease categories in FS ( $p=0.050)$; between group $1-3, p=0.005$. Conclusions: As expected, FS measured by PKG increase during disease progression in PD. In advanced disease stages, FS stabilise, indicating that PKG is the most useful in early and moderate stages of PD.
\end{abstract}

\section{Keywords}

Late Stage Parkinson Disease, Levodopa, Disease Duration, Fluctuations, Dyskinesia, Bradykinesia

\section{Introduction}

Motor fluctuations (FS) are frequently seen during progression in Parkinson Disease (PD) patients [1] [2]. Therapeutic interventions are focused on minimising FS [3] [4]. The degree of motor symptoms combined with the degree of 
disability experienced by patients determines therapeutic decisions. This decision is based on the amount of disturbance/disability the patient is experiencing, but an effective monitoring of these complications is missing.

The severity of these FS used to be measured by clinical rating scales or diaries [3]. However, these methods have numerous limitations, including inter-rater subjectivity and inability to measure the motor state of the patient continuously [3] [4].

Recently, the Parkinson's KinetiGraph (PKG) has been developed to measure bradykinesia (BKS), dyskinesia (DKS) and FS almost continuously [3]. The aim of this study is to investigate whether the assessment of FS measured by PKG, is correlated with disease duration.

\section{Material and Methods}

The PKG is a method that collects movement data by an accelerometer placed around the wrist of the most severely affected side of PD patients [3]. Individual scores of BKS, DKS and as a result FS are being calculated [5].

The sample size consists of data from all PD patients with a successful PKG measurement (between 2014 and 2016) at the Medical Centre Leeuwarden (MCL), Martini Hospital Groningen and Isala Clinics Zwolle. Approval for this study is been given by the Medical Ethics Committee of MCL, Martini Hospital and Isala Clinics. PD patients wore the PKG at least 6 days. Primary study parameters are BKS and DKS and FS: The BKS is a quantitative measure of the bradykinesia a patient is experiencing during the day and is derived by calculating the maximum acceleration for each 2 minute period and the Mean Spectral Power (MSP) surrounding this peak. The algorithm recognises bradykinesia as having fewer movements, which are made with lower acceleration and amplitude and with longer intervals between movements, compared to normal subjects. The DKS is a quantitative measure of the dyskinesia a patient is experiencing during the day and is derived by calculating the mean acceleration and the MSP in a 2 min epoch. The algorithm recognises dyskinesia as having movements of normal amplitude and acceleration but with shorter periods without movement. The FS is a quantitative measure of the fluctuations a patient can experience during the day. The FS can be derived by summing the interquartile range of bradykinesia scores and dyskinesia scores and expressing this value as a logarithm. This score can be useful to identify patients whose fluctuations are progressing and who may require therapeutic changes [3] [5].

The renewed MDS PD diagnostic criteria were used. The essential criterion is Parkinsonism (defined as bradykinesia with rest tremor or rigidity) and once this has been established, a distinction can be made between clinically established and clinically probable PD. In clinically established PD, the specificity is maximized and the sensitivity is reduced whereas in clinically probable PD the specificity and sensitivity are balanced. The determination of PD relies on three categories: supportive criteria (positive features that increase the probability of 
PD), absolute exclusion criteria (which rule out PD) and red flags (which must be counterbalanced by supportive criteria to allow PD diagnosis). The exact diagnostic criteria are shown in Postuma et al. [6].

The exclusion criteria are defined as follows: use of advanced therapy (Deep Brain Stimulation (DBS), Apomorphine pump or duodopa treatment), restricted movement (e.g. wheelchair or bedbound), concomitant disease that interferes with mobility in addition to PD (e.g. arthritis and rheumatisms) or PKG not worn for a minimum of 6 days.

This is a multicentre, retrospective, observational study, linking PKG outcome measures with clinical data. The following clinical data are collected from dossiers: sex, age, Parkinson's disease duration, mobility, number of levodopa doses per day, levodopa equivalent dose and other (motor) details regarding PD.

IBM SPSS Statistics 19 was used for all statistical analyses. Descriptive analyses were used to describe patient characteristics by means with standard deviation and medians with minimal and maximal values. Categorical variables were described by frequencies with percentages. The variable disease duration is, based on percentiles for equal groups, categorized into four categories: 0 - 5 years, 6 - 7 years, 8 - 11 years and $\geq 12$ years. Based on these categories, the variables are further described. The relation between disease duration and the variables levodopa equivalent dose, BKS, DKS and FS was calculated by Kruskal-Wallis test for independent samples with a significance set at $\mathrm{p} \leq 0.05$.

The relation between disease duration and the variables age, sex, levodopa times/day, use of extra dopaminergic medication and use of walking aid was calculated with a chi-square test. For variables with significant differences between categories, the Mann-Whitney $\mathrm{U}$ was used to calculate the deviating group.

\section{Results}

In total, data of 215 patients who were measured in the period of December 2014 to May 2016 were collected. Of these 215 patients, 18 patients were measured twice and 3 patients were measured three times resulting in a total of 239 measurements. Of these patients, 41 patients with in total 48 measurements met the exclusion criteria and were excluded. Therefore, the statistical analysis is performed on 174 patients with a total of 191 measurements. Of one patient, the disease duration was not known. Table 1 presents the patient characteristics. Results of the analysis of the variables, subdivided by disease duration are shown in Table 2.

A tendency for a reverse parabolic configuration was found in the FS: a deflecting rising curve between the first three categories and a descending curve between the third and fourth category. There was a significant difference found between the categories $(p=0.050)$. A significant increase in FS was found between the first and third category $(\mathrm{p}=0.005)$, followed by a slight decrease in the fourth category.

The following significant results were found between the categories: 
Table 1. Patient characteristics.

\begin{tabular}{|c|c|}
\hline Age (years), mean $( \pm S D)$ & \\
\hline Male & $68.7(8.82)$ \\
\hline Female & $69.7(7.27)$ \\
\hline \multicolumn{2}{|l|}{ Sex, n (\%) } \\
\hline Male & $102(53 \%)^{*}$ \\
\hline Female & $89(47 \%)$ \\
\hline \multicolumn{2}{|l|}{ Disease duration (years), median (min-max) } \\
\hline Male & $7(1-28)$ \\
\hline Female & $8(1-21)$ \\
\hline Missing & $\mathrm{n}=1$ \\
\hline \multicolumn{2}{|l|}{ Walking aid, n (\%) } \\
\hline None & $145(75.9 \%)$ \\
\hline Walking sticks & $4(2.1 \%)$ \\
\hline Crutches & $2(1.0 \%)$ \\
\hline Walker & $36(18.8 \%)$ \\
\hline Mobility scooter & $1(0.5 \%)$ \\
\hline Combination & $3(1.6 \%)$ \\
\hline Levodopa equivalent dose (mg), median (min-max) & $700(0-2100)$ \\
\hline Male & $800(0-2100)$ \\
\hline Female & $650(100-1350)$ \\
\hline \multicolumn{2}{|l|}{ Use of other anti-Parkinson medication, $\mathrm{n}(\%)$} \\
\hline No extra drugs & $60(31.4 \%)$ \\
\hline 1 extra drug & $97(50.8 \%)$ \\
\hline 2 extra drugs & $31(16.2 \%)$ \\
\hline 3 extra drugs & $2(1.0 \%)$ \\
\hline 4 extra drugs & $1(0.5 \%)$ \\
\hline
\end{tabular}

${ }^{*}$ For statistical analysis see Table 2 .

Table 2. Disease duration divided in four categories.

\begin{tabular}{|c|c|c|c|c|c|}
\hline \multirow[b]{2}{*}{ Category } & \multicolumn{5}{|c|}{ Disease duration } \\
\hline & $1(0-5$ years $)$ & $2(6-7$ years $)$ & $3(8-11$ years $)$ & 4 ( $\geq 12$ years $)$ & $\mathrm{p}$ \\
\hline Age, median (min-max) & $70(48-87)$ & $70(46-88)$ & $67(47-82)$ & $73(56-87)$ & $0.540^{\mathrm{b}}$ \\
\hline Sex, n (male) & $43(21)$ & $51(31)$ & $51(25)$ & $45(25)$ & $0.583^{\mathrm{b}}$ \\
\hline Levodopa equivalent dose (mg), median (min-max) & $600(0-1500)$ & $700(300-1500)$ & $800(150-1650)$ & $750(375-2100)$ & $0.078^{\mathrm{a}}$ \\
\hline Levodopa dose times/day, median (min-max) & $4(0-8)$ & $5(3-10)$ & $5(3-8)$ & $5(3-10)$ & $0.006^{* \mathrm{~b}}$ \\
\hline Extra anti-Parkinson medication use, $\mathrm{n}(\%)$ & $23(53.5 \%)$ & $33(64.7 \%)$ & $39(76.5 \%)$ & $35(77.8 \%)$ & $0.044^{* \mathrm{~b}}$ \\
\hline Use of walking aid, n (\%) & $7(16.3 \%)$ & $8(15.7 \%)$ & $13(25.5 \%)$ & $18(40.0 \%)$ & $0.022^{* \mathrm{~b}}$ \\
\hline Bradykinesia, median (min-max) & $26.40(11.30-42.60)$ & $24.90(15.90-43.70)$ & $25.30(12.60-45.30)$ & $24.70(13.70-51.95)$ & $0.877^{\mathrm{a}}$ \\
\hline Dyskinesia, median (min-max) & $1.10(0.20-26.85)$ & $2.20(0.10-27.00)$ & $2.40(0.10-25.80)$ & $2.10(0.10-31.90)$ & $0.094^{\mathrm{a}}$ \\
\hline Fluctuation score, median (min-max) & $8.00(4.10-25.10)$ & $9.20(4.50-25.40)$ & $9.30(5.40-12.30)$ & $9.10(4.40-22.80)$ & $0.050^{\star a}$ \\
\hline
\end{tabular}

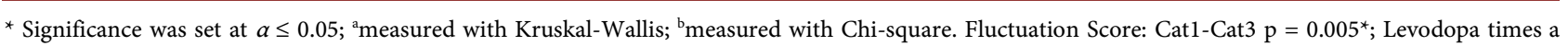
day: Cat1-Cat2 $\mathrm{p}=0.005^{*}$; Cat1-Cat3 $\mathrm{p}<0.000^{*}$; Cat1-Cat4 $\mathrm{p}<0.000^{*}$; Cat2-Cat4 $\mathrm{p}=0.042^{*}$; Anti-Parkinson medication use: Cat1-Cat3 $\mathrm{p}=0.020^{*}$; Cat1-Cat $4 \mathrm{p}=0.017^{*}$; Use of walking aid: Cat1-Cat $4 \mathrm{p}=0.014^{*}$; Cat2-Cat $4 \mathrm{p}=0.008^{*}$. 
- Levodopa times a day $(\mathrm{p}=0.006)$; group $1-2: \mathrm{p}=0.005$; group $1-3: \mathrm{p}=$ 0.000; group $1-4: \mathrm{p}=0.000$ ); group $2-4: \mathrm{p}=0.042$.

- Extra-Parkinson medication $(\mathrm{p}=0.044)$; group $1-3: \mathrm{p}=0.020$; group $1-4$ : $\mathrm{p}$ $=0.017$.

- Use of walking aid ( $\mathrm{p}=0.022)$; group $1-4: \mathrm{p}=0.014$; group $2-4: \mathrm{p}=0.008$.

\section{Discussion}

Using the PKG, a significant increase in FS was found during disease progression in PD patients. This is in accordance with several studies that found a positive relation in frequency between motor complications (FS and dyskinesias) and disease progression (set by clinical rating scales) [7]. However, in our study, FS tend to decrease in the most advanced stages of PD. This is in line with previous studies which described a spontaneous "resolution" or a "remission" of dyskinesia and FS during the later or end stages of $\mathrm{PD}$, without any reduction in levodopa treatment and/or in the dose of anti-Parkinson drugs [8] [9]. Papapetropoulos et al. mentioned a decrease in dopaminergic receptor density and post-synaptic degeneration with disease progression as a possible explanation for the resolution [8]. We have to take in consideration, that late stage PD patients use significant more walking aid. This could be an explanation for a decline in FS in later stages of PD. When patients use a walker, the wrist is more stable than when walking without. This fixation can have a negative influence on the primary variables.

Surprisingly, no difference in BKS between groups was found. As a result, FS are completely dependent by DKS. We had expected to find lower BKS early in the course of the disease. Possibly, an effective BKS treatment was set in all groups, or the occurrence of DKS was more pronounced in later stages compared to the early stages. Another explanation can be that the PKG is not sensitive enough to measure this difference in BKS.

As expected, the distribution of levodopa intake (times a day) was different between the categories [10]. In later stages of $\mathrm{PD}$, levodopa is more frequently used with shorter intervals. Although, not significant, a tendency for increased amounts of levodopa was seen during disease progression, probably to minimise FS [7]. However, in the most advanced PD patients, a slightly decrease in levodopa amount was seen, suggesting that the maximum beneficial level of levodopa was reached. Probably, using supra-maximal levodopa, minimal response was reached and more adverse effects were present [9] [11] [12] [13]. On the other hand, other Parkinson medications were more used in later phases of PD.

Several factors in our study might have influenced the results. Firstly, we have a relatively small group of patients. Secondly, all patients were already measured in the context of therapy, so we could benefit and include many patients in a short period of time. However, this method created a selection bias. The clinicians selected patients for the PKG registration, because their FS were not optimally controlled by their present medication. This results in a study population 
with only poorly controlled patients. Additionally, no control study group was taken in our design. Thirdly, the available data we could abstract from the medical dossiers was limited due to the study design. To further investigate the evolution of motor complications with the progression of the disease, a large prospective cohort study can be performed. In conclusion, in the early and middle phase of Parkinson disease, an increase of FS is seen. In advanced stages (more than 12 years of disease) FS stabilise.

\section{References}

[1] Kalia, L.V. and Lang, A.E. (2015) Parkinson's Disease. Lancet, 386, 896-912. https://doi.org/10.1016/S0140-6736(14)61393-3

[2] Nutt, J.G. (2008) Pharmacokinetics and Pharmacodynamics of Levodopa. Movement Disorders, 23, S580-S584. https://doi.org/10.1002/mds.22037

[3] Griffiths, R.I., Kotschet, K., Arfon, S., et al. (2012) Automated Assessment of Bradykinesia and Dyskinesia in Parkinson's Disease. J Parkinsons Dis, 2, 47-55.

[4] Bergquist, F. and Horne, M. (2014) Can Objective Measurements Improve Treatment Outcomes in Parkinson's Disease? European Neurological Review, 9, 27-30. https://doi.org/10.17925/ENR.2014.09.01.27

[5] Horne, M.K., McGregor, S. and Bergquist, F. (2015) An Objective Fluctuation Score for Parkinson's Disease. PLoS ONE, 10, e0124522. https://doi.org/10.1371/journal.pone.0124522

[6] Postuma, R.B., Berg, D., Stern, M., et al. (2015) MDS Clinical Diagnostic Criteria for Parkinson's Disease. Movement Disorders, 30, 1591-1599.

https://doi.org/10.1002/mds.26424

[7] Kempster, P.A., Williams, D.R., Selikhova, M., Holton, J., Revesz, T. and Lees, A.J. (2007) Patterns of Levodopa Response in Parkinson's Disease: A Clinico-Pathological Study. Brain, 130, 2123-2128. https://doi.org/10.1093/brain/awm142

[8] Papapetropoulos, S. and Mash, D.C. (2007) Motor Fluctuations and Dyskinesias in Advanced/End Stage Parkinson's Disease: A Study from a Population of Brain Donors. Journal of Neural Transmission, 114, 341-345. https://doi.org/10.1007/s00702-006-0603-6

[9] Coelho, M., Marti, M.J., Tolosa, E., et al. (2010) Late-Stage Parkinson's Disease: The Barcelona and Lisbon Cohort. Journal of Neurology, 257, 1524-1532. https://doi.org/10.1007/s00415-010-5566-8

[10] Connolly, B.S. and Lang, A.E. (2014) Pharmacological Treatment of Parkinson Disease. A Review. JAMA, 311, 1670-1683. https://doi.org/10.1001/jama.2014.3654

[11] Fabbri, M., Coelho, M., Abreu, D., et al. (2016) Do Patients with Late-Stage Parkinson's Disease Still Respond to Levodopa? Parkinsonism \& Related Disorders, 26, 10 16. https://doi.org/10.1016/j.parkreldis.2016.02.021

[12] Coelho, M., Marti, M.J., Sampaio, C., et al. (2015) Dementia and Severity of Parkinsonism Determines the Handicap of Patients in Late-Stage Parkinson's Disease: The Barcelona-Lisbon Cohort. European Journal of Neurology, 22, 305-312. https://doi.org/10.1111/ene.12567

[13] Hand, A., Gray, W.K., Oates, L.L., et al. (2016) Medication Use in People with Late Stage Parkinson's Disease and Parkinsonism Living at Home and in Institutional Care in North-East England: A Balance of Symptoms and Side-Effects? Parkinsonism \& Related Disorders, 32, 120-123. https://doi.org/10.1016/j.parkreldis.2016.09.001 